\title{
PRODUÇÃO DE BIOMASSA DE CANA-DE-AÇÚCAR EM DOIS ESPAÇAMENTOS E DUAS FREQUÊNCIAS DE CORTES ${ }^{1}$
}

\author{
Forage yield of sugarcane growing in different row spacing and harvesting periods ${ }^{1}$
}

\author{
Gisele Bonato Muraro 2 , Paulo Rossi Junior³, Ana Luiza Bachmann Schogor ${ }^{4}$
}

\begin{abstract}
RESUMO
Objetivou-se, neste trabalho, identificar os efeitos do espaçamento de plantio de $0,90 \mathrm{~m}$ e 1,30 $\mathrm{m}$ e da frequência de um ou dois cortes na produção de biomassa da cana-de-açúcar. O experimento foi realizado em três fazendas no município de Santo Antônio da Platina - PR, utilizando-se a cultivar RB72454 implantada em novembro de 2004. As avaliações foram realizadas até janeiro de 2006. Os tratamentos foram compostos da combinação dos espaçamentos de $0,90 \mathrm{~m}$ e $1,30 \mathrm{~m}$ com as frequências de um ou dois cortes, aos 240 dias cana-planta e 180 dias cana-soca ou 420 dias de cana-planta, respectivamente. O delineamento experimental utilizado foi de blocos casualizados em esquema fatorial 2x2 e cinco repetições. A produção de biomassa da cultivar RB72454 variou entre 142,49 t-ha-1 e 225,70 t-ha ${ }^{-1}$ e foi resultante do efeito do espaçamento de plantio. A frequência de cortes afetou $(\mathrm{P}<0,05)$ a produtividade, sendo que a frequência de 1 corte foi mais produtiva em $21,41 \%$ que em 2 cortes. A perda relativa a tocos em situação de colheita mecanizada representou, em média, $17,41 \%$ da produtividade total para a frequência de 2 cortes e $6,83 \%$ para frequência de 1 corte.
\end{abstract}

Termos para indexação: Acúmulo de massa seca, forragem, população de plantas, Saccharum spp.

\section{ABSTRACT}

The objective of this study was to identify the effect of $0.90 \mathrm{~m}$ and $1.30 \mathrm{~m}$ row spacing and one or two periods of harvesting on the agronomical characteristics and forage yield of sugarcane cultivar RB72454. The trial was performed in three farms located in Santo Antônio da Platina - PR, from November 2004 to January 2006. Treatments were composed by combination of $0.90 \mathrm{~m}$ and $1.30 \mathrm{~m}$ row spacing and number of harvesting, being two periods of harvesting (240 days of plant-cane and 180 days of regrowth), or one harvesting (420 days of plant-cane). A randomized blocks design was used, with five replicates per treatment. The yield forage varied between 142.49 t-ha $^{-1}$ and 225.70 t-ha $^{-1}$ and was due to row spacing. The period of harvesting affected $(\mathrm{P}<0.05)$ the yield and the treatment of one cut was $21.41 \%$ more productive than two harvests. The stubbles losses in simulated mechanical harvesting represented, on average, $17.41 \%$ and $6.83 \%$ of the total yield for two and one harvesting, respectively.

Index terms: Accumulation of dry matter, forage, plant population, Saccharum spp.

(Recebido em 27 de fevereiro de 2008 e aprovado em 20 de agosto de 2008)

\section{INTRODUÇÃO}

O uso da cana-de-açúcar na alimentação de bovinos tem importância cada vez maior em virtude do menor custo da cultura, substituindo silagem de milho e de sorgo. A principal restrição tem sido a mecanização do corte, porque as máquinas nacionais utilizadas para colheita são precárias, de baixo rendimento e tamanho de corte de partículas não adequado ao bom desempenho animal (Balsalobre et al., 1999).

O sucesso da utilização da cana-de-açúcar na alimentação de ruminantes deve-se a sua alta produtividade de matéria seca por área, tradição no cultivo e baixos custos. A produtividade agrícola da cultura está sujeita a interferências do ambiente em que está instalada, promovendo diferentes resultados nas plantas de acordo com o estádio fenológico em que se encontram. Além das condições ambientais, como clima e solo, manejo agrícola, variedade, idade da muda e estádio de desenvolvimento da cultura também influenciam na produtividade.

Trabalhos pioneiros com redução de espaçamento entrelinhas revelaram tendência a aumento de produtividade da cultura de cana-de-açúcar. Stubbs (1895), citado por Galvani et al. (1997) conduziu experimentos precursores com cana-de-açúcar plantada em espaçamentos de 1,50 e 0,90 m entrelinhas obtendo resultados de aumento de produtividade, em cana-planta e cana-soca, de $23 \%$. Também vários pesquisadores confirmam aumento de produtividade com adoção de espaçamentos de plantio reduzidos (Espironelo et al., 1987; Galvani et al., 1997).

\footnotetext{
${ }^{1}$ Parte da Dissertação de Mestrado em Ciências Veterinária, apresentada pela primeira autora à Universidade Federal do Paraná/UFPR 2Universidade de São Paulo/USP - Escola Superior de Agricultura Luiz de Queiroz/ESALQ - Piracicaba, SP - In memorian ${ }^{3}$ Universidade Federal do Paraná/UFPR - Departamento de Zootecnia/DZ - Rua dos Funcionários - 1540 - Juvevê - 80035-050 - Curitiba, PR parossi@ufpr.br

${ }^{4}$ Universidade de São Paulo/USP - Escola Superior de Agricultura Luiz de Queiroz/ESALQ - Piracicaba, SP
} 
Dados de produtividade de cana-de-açúcar são reportados na literatura, em sua maioria, na fase de maturação da cultura, entretanto o acompanhamento do rendimento forrageiro e acúmulo de matéria seca são importantes em sistemas que desejam utilizar esse material forrageiro buscando otimizar o retorno econômico da cultura.

A cana-de-açúcar, quando cultivada para o corte mecanizado, deve ser adaptada para tal atividade. Devido às limitações das máquinas para o corte da cana-de-açúcar para forragem, a cultura deve ter algumas características consideradas prioritárias para o sucesso desse sistema de produção. A cultivar ideal para o corte mecanizado deve possuir menor número de colmos na linha de plantio, alta produtividade e o porte ereto (não estar tombada, acamada).

Objetivou-se, neste trabalho, avaliar o rendimento forrageiro da cana-de-açúcar, cultivar RB72454, plantada em dois espaçamentos e submetida a duas frequências de corte no período de 420 dias.

\section{MATERIAL E MÉTODOS}

Os trabalhos foram conduzidos em três propriedades localizadas no município de Santo Antônio da Platina no estado do Paraná. A área experimental constou de dois talhões de 1 ha cada, em cada uma das propriedades. A área foi delimitada respeitando uma bordadura de 5 linhas de plantio em cada lado do talhão. As áreas escolhidas estavam sob mesma condição de relevo, com mesmos tratos culturais e de adubação e estão localizadas em um raio de aproximadamente $5 \mathrm{~km}$.

O clima da região, segundo a classificação de Koppen, é Cfa, subtropical. O solo é classificado como argissolo vermelho de relevo levemente ondulado. $\mathrm{O}$ plantio deu-se em novembro de 2004, e com a distribuição das mudas utilizou-se a densidade média de 15 gemas por metro de sulco para ambos os espaçamentos, no sistema "pé com ponta", com rebolos de 3 gemas. Para a adubação de plantio utilizou-se $60 \mathrm{~kg}$ de $\mathrm{P}_{2} \mathrm{O}_{5}$ e $100 \mathrm{~kg}$ de $\mathrm{KCl}$ por hectare acrescida de adubação de cobertura com $45 \mathrm{~kg}$ de nitrogênio por hectare, em fevereiro de 2005. No talhão correspondente à frequência de 2 cortes foi feita nova adubação de cobertura com $45 \mathrm{~kg}$ de nitrogênio e $45 \mathrm{~kg}$ $\mathrm{KCl}$, em outubro de 2005 .

Duas semanas após o plantio foi realizado o controle de plantas daninhas com herbicidas para folhas largas e gramíneas e repetido em janeiro de 2005 e, para o talhão correspondente à rotina de 2 cortes, foi realizada novamente em outubro de 2005.
O plantio, adubação e tratos culturais foram realizados simultaneamente nas três fazendas. O delineamento experimental utilizado foi o de blocos casualizados, em esquema fatorial $2 \times 2$ e cinco repetições. $\mathrm{O}$ primeiro fator correspondeu aos espaçamentos de plantio entrelinhas de $0,90 \mathrm{~m}$ (reduzido) e 1,30 m (tradicional). $\mathrm{O}$ segundo fator correspondeu à frequência 1 ou 2 cortes, no período de 420 dias. No caso da frequência de 2 cortes, o primeiro foi realizado quando a cana-deaçúcar estava com 240 dias de crescimento e o segundo corte quando possuía 180 dias de crescimento (rebrota).

As avaliações de produtividade foram realizadas em 10 metros de cinco linhas consecutivas de plantio. $\mathrm{O}$ início de uma linha escolhida para avaliação correspondia ao final da linha adjacente, permitindo assim uma avaliação mais distribuída em toda a extensão do talhão, totalizando a amostragem em 50 metros lineares por espaçamento de plantio.

O número de perfilhos foi avaliado em 1 metro de cada linha de plantio, contando-se os perfilhos. Em seguida, 20 perfilhos em cada linha foram cortados manualmente, formando um feixe correspondente a cada linha de plantio. Foram mensurados os sólidos solúveis ( ${ }^{\circ}$ brix) por meio de um refratômetro de campo (marca TOKYO $^{\circledR}$ modelo 032), em 5 perfilhos, na base, meio e ponta, gerando posteriormente a média do colmo.

Foram obtidas as massas dos feixes e em seguida $20 \mathrm{~cm}$ da base do perfilho (tocos) foram cortados, pesados e subtraídos da fração do feixe para a simulação de perdas no campo em situações de colheita mecanizada.

Para 2 frequências de cortes $\left(\mathrm{t}-\mathrm{ha}^{-1}\right)$ obteve-se: produção de material fresco (colmo + folhas), produção de material seco e de toco, obtidos por meio da soma dessas partes no $1^{\circ}$ e $2^{\circ}$ corte; enquanto que para sólidos solúveis ( ${ }^{\circ}$ brix), número de perfilhos e massa média do perfilho, os dados são apresentados na forma de média aritmética do $1^{\circ}$ e $2^{\circ}$ corte.

A massa média do perfilho foi calculada dividindose a massa do feixe pelo número de perfilhos.

$\mathrm{O}$ cálculo de produtividade de material fresco $\left(\mathrm{t}-\mathrm{ha}^{-1}\right)$ foi feito por meio da multiplicação do número de colmos por metro pelo fator de 11.111, para o espaçamento de $0,90 \mathrm{~m}$, e pelo fator 7.692 para o espaçamento de $1,30 \mathrm{~m}$. Esses fatores de multiplicação referem-se à metragem linear teórica por hectare.

A produtividade em material seco (colmo + folhas) foi calculada a partir da produtividade do material fresco multiplicado pelo teor de matéria seca. $\mathrm{O}$ teor de matéria seca foi calculado a partir do peso do material após secagem em estufa de ar forçado à temperatura de $60^{\circ} \mathrm{C}$, por 48 horas. 
Os dados experimentais foram analisados estatisticamente pelos procedimentos de análise de variância, por meio do programa STATISTICA 6.0. Para efeito de comparação de médias, foi utilizado o teste de Tukey, a 5\% de probabilidade.

\section{RESULTADOS E DISCUSSÃO}

Os dados de produção de biomassa da cana-deaçúcar nos diferentes espaçamentos e frequência de corte, encontram-se na Tabela 1. A produtividade de material fresco obtido esteve entre 225,70 t-ha ${ }^{-1}$ e 142,49 t-ha ${ }^{-1}$, referentes aos tratamentos de 1 corte no espaçamento de $0,90 \mathrm{~m}$ e 2 cortes no espaçamento de $1,30 \mathrm{~m}$, respectivamente.

Os valores de produção de biomassa fresca obtidos neste trabalho estão próximos aos obtidos por França et al. (2006) de 205,67 t-ha-1 de cana-de-açúcar RB72454 irrigada e colhida aos 450 dias. Rocha et al. (1998) testando 6 cultivares de cana-de-açúcar obtiveram média de 144,6 t-ha-1 e a cultivar RB72454 se destacou com produção de 178,9 t-ha-1 em espaçamento de 1,40 m.

Apesar da alta produtividade obtida, principalmente para frequência de 1 corte, deve-se salientar que a cultura sofreu período de restrição hídrica aos 3 meses de desenvolvimento, fato que pode ter comprometido o acúmulo de massa verde, com efeito mais significativo para o $1^{\circ}$ corte da cana-de-açúcar, submetida ao tratamento de 2 frequências de corte. Segundo Ramesh \& Mahadevaswamy (2000) a ocorrência de déficit hídrico que proporciona maiores diminuições nas produtividades de cana planta é no período de máximo desenvolvimento e na cana-soca no estádio inicial de crescimento.
Neste estudo verificou-se que a produção de material fresco foi significativamente afetada pela frequência de corte e espaçamento. $\mathrm{O}$ peso médio de perfilhos combinados com frequência de 1 corte nos dois espaçamentos testados resultaram em maiores acúmulos de biomassa. Além disso, observa-se que a redução do espaçamento incrementou a produtividade, principalmente para 2 cortes, com aumento de $22 \%$ contra $16 \%$ para 1 corte, representando 40,93 t-ha' ${ }^{-1}$.

$\mathrm{O}$ incremento da produtividade relacionada com a redução do espaçamento também foi observada por Espironelo et al. (1987) da ordem de 14\% em latossolo roxo e $7 \%$ em latossolo vermelho escuro, quando houve redução de 1,50 m para 1,20 m. No entanto, Galvani et al. (1997) relatam acréscimos de $9 \%$ com a redução do espaçamento de $1,80 \mathrm{~m}$ para $0,90 \mathrm{~m}$, representando um aumento de 9,24 t-ha-1 de cana-de-açúcar.

Ao se considerar a produção de material seco observa-se diferença significativa $(\mathrm{P}<0,05)$ entre a freqüência de cortes, sendo que as maiores produções obtidas são para 1 corte, com valores de 56,67 t-ha $^{-1}$ para espaçamento de $0,90 \mathrm{~m} \mathrm{e} 48,12 \mathrm{t}$-ha ${ }^{-1}$ para o espaçamento de $1,30 \mathrm{~m}$. O tratamento de 1 corte resultou em maior rendimento de material seco em função da oportunidade para acúmulo de biomassa e do acúmulo de carboidratos solúveis no colmo, uma vez que as plantas submetidas a duas frequências de corte foram colhidas antes da fase final de crescimento. $\mathrm{O}$ efeito do espaçamento de plantio foi significativo $(\mathrm{P}<0,05)$ para 1 corte, com maior produção obtida para o espaçamento de $0,90 \mathrm{~m}$, reflexo da maior produtividade de material fresco obtida nesse espaçamento.

Tabela 1 - Produção de material fresco, material seco, número de perfilhos, peso médio dos colmos, \% toco e sólidos solúveis da cultivar RB72454; obtidos em função dos tratamentos aplicados.

\begin{tabular}{|c|c|c|c|c|c|}
\hline \multirow{3}{*}{ Variáveis } & \multicolumn{4}{|c|}{ Tratamentos } & \multirow{3}{*}{$\mathrm{CV}(\%)$} \\
\hline & \multicolumn{2}{|c|}{$0,90 \mathrm{~m}$} & \multicolumn{2}{|c|}{$1,30 \mathrm{~m}$} & \\
\hline & 1 corte & 2 cortes & 1 corte & 2 cortes & \\
\hline Produção de material fresco $\left(\mathrm{t}-\mathrm{ha}^{-1}\right)$ & $225,70 \mathrm{a}$ & $183,42 \mathrm{~b}$ & $189,01 \mathrm{~b}$ & $142,49 \mathrm{c}$ & 22 \\
\hline Produção de material seco $\left(\mathrm{t}-\mathrm{ha}^{-1}\right)$ & 56,67 a & $34,43 \mathrm{c}$ & $48,12 \mathrm{~b}$ & $27,83 \mathrm{c}$ & 21 \\
\hline Número de perfilhos por metro linear & $12,87 \mathrm{~b}$ & $17,10 \mathrm{a}$ & $13,73 \mathrm{~b}$ & $17,40 \mathrm{a}$ & 22 \\
\hline Peso médio de perfilho (kg) & $1,62 \mathrm{~b}$ & $0,48 \mathrm{c}$ & $1,86 \mathrm{a}$ & $0,54 \mathrm{c}$ & 19 \\
\hline Toco, $\mathrm{t}^{-h a^{-1}}$ & $16,74 \mathrm{c}$ & $31,27 \mathrm{a}$ & $11,79 \mathrm{~d}$ & $25,33 \mathrm{~b}$ & 29 \\
\hline \% Toco da produção de material fresco & $7,42 \mathrm{~b}$ & $17,05 \mathrm{a}$ & $6,24 \mathrm{~b}$ & $17,78 \mathrm{a}$ & 20 \\
\hline Sólidos solúveis ( ${ }^{\circ}$ brix) & $16,87 \mathrm{a}$ & $8,24 \mathrm{~b}$ & $17,45 \mathrm{a}$ & $8,88 \mathrm{~b}$ & 19 \\
\hline
\end{tabular}

Médias seguidas de letras diferentes nas linhas, diferem entre si $(\mathrm{P}<0,05)$; pelo teste de Tukey. 
Os valores apresentados por Andrade et al. (2003), que resultam de compilação de avaliação de 39 cultivares de cana-de-açúcar, para alimentação animal, são da ordem de 20,98 t-ha-1 a 53,86 t-ha-1. Rocha et al. (1998) apresentam produtividade de 42,2 t-ha- ${ }^{-1}$ avaliando cana-de-açúcar aos 330 dias de crescimento.

As diferenças encontradas para produção de material seco nos diversos trabalhos apresentados estão correlacionados com o teor de matéria seca característico das cultivares testadas, bem como da época de colheita. Como observado no trabalho desenvolvido por Andrade et al. (2003), o teor de matéria seca da cana-de-açúcar colhida aos 12 meses foi superior ao da cana-de-açúcar colhida aos 18 meses, devido ao período de seca antes da colheita.

Analisando os valores do número de perfilhos, observa-se que a frequência de 2 cortes resultou em maior perfilhamento, devido ao fato de que a cana-de-açúcar colhida aos 240 e 180 dias estava na fase de intenso perfilhamento, com efeito pronunciado no corte aos 240 dias. Efeito também observado no estudo de Oliveira et al. (2007) que, para a mesma variedade deste estudo, constatou perfilhamento máximo próximo aos 231 dias após o plantio. A partir desta etapa a competição entre os perfilhos pelos fatores de crescimento (luz, água, nutrientes, espaço) acentua-se, de modo que se constata a diminuição e paralisação deste processo (Segato et al., 2006), como pode ser observado para cana-de-açúcar de 1 corte, que representa a planta no final do estádio de maturação.

No presente estudo, o número médio foi de 13,3 perfilhos/metro para a frequência de 1 corte. Os resultados estão de acordo com as características da cultivar RB72454, expostas por Landell et al. (2002) que relatam 11,47 perfilhos/metro em espaçamento de $1,30 \mathrm{~m}$ em cultura avaliada em estádio de maturação; e de acordo com França et al. (2006) que relatam número de 10,32 perfilhos/metro, avaliando a mesma variedade, com espaçamento de 1,30 m e corte aos 450 dias de idade.

Considerando-se a fase de desenvolvimento vegetativo, presente para a frequência de 2 cortes, os dados deste trabalho estão acima dos obtidos por Oliveira et al. (2004), que trabalharam com a mesma cultivar, em nove épocas de desenvolvimento e obtiveram 13,6 perfilhos/m linear aos 182 dias e 9,4 perfilhos/m linear para 428 dias. Rocha et al. (1998) obtiveram números superiores aos relatados, porém próximos a este estudo, de 17,5 perfilhos/ m para cana-de-açúcar com 240 dias de idade.

A massa média dos perfilhos apresentou efeito significativo $(\mathrm{P}<0,05)$ do espaçamento para frequência de 1 corte. A maior massa média dos perfilhos referentes a 1 corte é resultante da oportunidade que as plantas tiveram para finalizar o acúmulo de matéria seca representado pelas folhas e colmo e pela deposição de sacarose atingindo valores médios de $1,62 \mathrm{~kg}$ e 1,86 $\mathrm{kg}(0,90 \mathrm{~m}$ e 1,30 m), assim como observado por Andrade et al. (2003), Moura et al. (2005) e Oliveira et al. (2007).

Os sólidos solúveis variaram significativamente $(\mathrm{P}<0,05)$ com a idade de corte e não tiveram efeito significativo do espaçamento. Os valores médios encontrados neste trabalho foram de 8,56 e 17,16 para a cana-de-açúcar de 2 e 1 corte, respectivamente. As magnitudes encontradas estão de acordo com os relatados por Rodrigues et al. (1997) de 17,8, porém abaixo do encontrado por Shigaki et al. (2004) 21,93, com cana cortada aos 330 dias. Para a cana de 2 cortes, os valores estão bem abaixo dos relatados por Rocha et al. (1998) de 15,1 e Banda \& Valdez (1976) de 14,5.

Cabe ressaltar que a cultivar utilizada neste trabalho é considerada de acúmulo de sacarose intermediária-tardia, sendo que o final do período de maturação dá-se nos meses de setembro - outubro, na região Centro-Sul do país. Neste trabalho, a cana-de-açúcar de frequência 1 corte foi colhida no período de janeiro, época característica de altos índices de precipitação. A maior disponibilidade de água é um estímulo para a planta reiniciar o desenvolvimento vegetativo, com utilização da energia presente na forma de sacarose. Além disso, a maior umidade presente na planta é um fator diluidor da sacarose que já estava acumulada nos internódios da planta.

A porcentagem de toco apresentou diferença significativa $(\mathrm{P}<0,05)$ para a frequência de cortes, independente do espaçamento. $\mathrm{O}$ toco que permanece após a colheita mecanizada é considerado uma perda visível e de acordo com esse estudo, pode representar $17,78 \%$ da produção total, em caso de 2 cortes e $7,42 \%$ para 1 corte na cana-planta. Cabe ressaltar que o presente estudo avaliou as perdas referentes a 1 corte e sugere-se que as perdas reduzam a medida que aumente o número de cortes, em virtude do rebaixamento natural da linha de plantio.

Neste trabalho, os valores da fração toco tiveram amplitude de 11,79 t-ha-1 a 31,27 t-ha ${ }^{-1}$, com perdas significativamente superiores para a frequência de 2 cortes. A única referência encontrada no sentido de perdas pela fração toco em canaviais forrageiros são de Balsalobre et al. (1999) que relatam que fazendas que fizeram a coleta de toco, após a colheita encontraram perdas de até $8 \mathrm{t}^{-h a^{-1}}$.

Os resultados de rendimento de material fresco e seco do feixe e do toco, nos diferentes cortes da cana-deaçúcar cultivar RB72454, estão apresentados na Tabela 2. 
Tabela 2 - Produção de material fresco e produção de material seco referente à fração feixe e fração toco da cana-deaçúcar cultivar RB72454, em função dos tratamentos aplicados.

\begin{tabular}{lccccc}
\hline \multirow{2}{*}{\begin{tabular}{l} 
Variáveis \\
\multicolumn{1}{c}{ t-ha }
\end{tabular}} & \multicolumn{2}{c}{$0,90 \mathrm{~m}$ Tratamentos } & \multicolumn{2}{c}{$1,30 \mathrm{~m}$} & CV (\%) \\
& 1 corte & 2 cortes & 1 corte & 2 cortes \\
\hline Produção de material fresco (feixe) & $209,01 \mathrm{a}$ & $152,36 \mathrm{~b}$ & $177,26 \mathrm{ab}$ & $117,66 \mathrm{c}$ & 23 \\
Produção de material seco (feixe) & $52,50 \mathrm{a}$ & $28,35 \mathrm{~b}$ & $45,14 \mathrm{a}$ & $22,71 \mathrm{~b}$ & 27 \\
Produção de material fresco (toco) & $17,26 \mathrm{c}$ & $31,06 \mathrm{a}$ & $11,75 \mathrm{~d}$ & $25,06 \mathrm{~b}$ & 29 \\
Produção de material seco (toco) & $4,31 \mathrm{~b}$ & $6,08 \mathrm{a}$ & $2,99 \mathrm{c}$ & $5,16 \mathrm{~b}$ & 29 \\
\hline
\end{tabular}

Médias seguidas de letras diferentes nas colunas, diferem entre si $(\mathrm{P}<0,05)$, pelo teste de Tukey.

A produção de material fresco do feixe apresentou diferença significativa entre as frequências de corte, no entanto, quando consideramos a produção em material seco, a diferença estatística $(\mathrm{P}<0,05)$ se apresenta para número de cortes, independente do espaçamento. A maior produção de material seco para frequência de 1 corte é reflexo da maior produção $\left(\mathrm{t}-\mathrm{ha}^{-1}\right)$ com maiores teores de matéria seca.

Observando a produção de material fresco relativo à fração do toco, houve diferença significativa $(\mathrm{P}<0,05)$ para frequência de cortes e para espaçamento. A produção de material seco do toco é relativa a \% de toco e o teor de matéria seca. A produção de material fresco do toco, neste estudo, representou $6,62 \%$ e 8,25 para 1 corte e $21,29 \%$ e $20,38 \%$ para 2 cortes, para espaçamento de $1,30 \mathrm{~m}$ e $0,90 \mathrm{~m}$, respectivamente. Considerando a produtividade de material seco, os porcentuais encontrados são de $6,62 \%$ a $8,25 \%$ para 1 corte e $22,72 \%$ a $21,44 \%$ para 2 cortes, para espaçamento de $1,30 \mathrm{~m}$ e $0,90 \mathrm{~m}$ respectivamente.

Pela fração toco apresentada na Tabela 2, indica-se a produção de material fresco e seco que poderia, potencialmente, ser pertencente à fração feixe em um sistema de colheita mais rente ao solo. A fração toco representou, neste trabalho, em material fresco e seco de $6,62 \%$ a $22 \%$ da fração feixe.

\section{CONCLUSÕES}

A redução do espaçamento entrelinhas de 1,30 m para $0,90 \mathrm{~m}$ foi efetiva no aumento do rendimento forrageiro da cultivar de cana-de-açúcar RB72454 e foi mais predominante para a frequência de 2 cortes que para 1 corte.

A perda pela fração toco resultou em média de $6,83 \%$ e $17,41 \%$ do rendimento total para a frequência de 1 e 2 cortes, respectivamente.

\section{AGRADECIMENTOS}

Aos proprietários das fazendas experimentais Marco Baggio Filho, Marco Baggio Netto e Nelson Simionatto, pela disponibilização das áreas experimentais, equipamentos e pessoal para a realização deste trabalho.

\section{REFERÊNCIAS BIBLIOGRÁFICAS}

ANDRADE, J.B.; FERRARI JUNIOR, E.; POSSENTI, R.A. Seleção de 39 variedades de cana-de-açúcar para alimentação animal. Brazilian of Journal Veterinary Research Animal Science, v.40, n.4, p.287-296, 2003.

BALSALOBRE, M.A.A.; SANTOS, P.M.; FERNANDES, R.A.T. Cana-de-açúcar: quando e como cortar para o consumo animal. Revista Balde Branco, São Paulo, n.421, p.19-13, 1999.

BANDA, M.; VALDEZ, R.E. Effect of stage of maturity on nutritive value of sugar cane. Tropical Animal Production, Tampico, v.1, n.2, p.94-97, 1976.

ESPIRONELO, A.; COSTA, A.A.; LANDELL, M.G.A.; PEREIRA, J.C.V.N.A.; IGUE,T.; CAMARGO, A.P.; RAMOS, M.T.B. Adubação NK em três variedades de cana-de-açúcar em função de dois espaçamentos.

Bragantia,Campinas, v.46, n.2, p.247-268, 1987.

FRANÇA, A.F.S.; MELLO, S.Q.S.; ROSA, B. Avaliação do potencial produtivo e das características químicobromatológicas de nove variedades de cana-de-açúcar irrigada. Livestock Research for Rural Development, v.17, n.7, 2006.

GALVANI, E.; BARBIERI, V.; PEREIRA, A.B. Efeitos de diferentes espaçamentos entre sulcos na produtividade agrícola da cana-de-açúcar (Saccharum spp.). Scientia Agricola, Piracicaba, v.54, n.1/2, p.62-68, 1997. 
LANDELL, M.G.A.; CAMPANA. M.P.; RODRIGUES, A.A. A Variedade IAC86-2480 como nova opção de canade-açúcar para fins forrageiros: manejo de produção e uso na alimentação animal. Campinas: IAC, 2002. 19p. (Boletim técnico, 193).

MOURA, M.V.P.S.; FARIAS, C.H.A.; AZEVEDO, C.A.V. Doses de adubação nitrogenada e potássica em cobertura na cultura da cana-de-açúcar, primeira soca, com e sem irrigação. Ciência e Agrotecnologia, Lavras, v.29, n.4, p.753-760, 2005.

OLIVEIRA, R.A.; DAROS, E.; ZAMBON, J.L.C. Área foliar em três cultivares de cana-de-açúcar e sua correlação com a produção de biomassa. Pesquisa Agropecuária Tropical, Goiânia, v.37, n.2, p.71-76, 2007.

OLIVEIRA, R.A.; DAROS, E.; ZAMBON, J.L.C. Crescimento e desenvolvimento de três cultivares de cana-de-açúcar, em cana-planta, no estado do Paraná. Scientia Agraria, Piracicaba, v.5, n.1/2, p.8794, 2004.

RAMESH, P.; MAHADEVASWAMY, M. Effect of formative phase drought on different classes of shoots, shoot mortality, cane attributes, yield and quality of four sugarcane cultivars. Journal of Agronomy and Crop Science, 185: 249-258, 2000.

ROCHA, R.; MIRANDA, M.; GONDIM, P.; ORTOLAN, A. Produtividade de cultivares de cana-de-açúcar no Oeste de Santa Catarina. Agropecuária Catarinense, Florianópolis, v.11, n.1, p.8-10, 1998.

RODRIGUES, A.A.; PRIMAVESI, O.; ESTEVES, S.N. Efeito da qualidade de variedades de cana-de-açúcar sobre seu valor como alimento para bovinos. Pesquisa Agropecuária Brasileira, Brasília, v.32, n.12, p.13331338, 1997.

SEGATO, S.V.; MATTIUZ, C.F.M.; MOZAMBANI, A.E. Aspectos fenológicos da cana-de-açúcar. In: SEGATO, S.V.; PINTO, A.S.; JENDIROBA, E. Atualização em produção de cana-de-açúcar. Piracicaba: Livroceres, 2006. p.19-36.

SHIGAKI, F.; BERTO, A.; LIMA, E.; ZONTA, E.; CEDDIA, M.B.; FREITAS, N. Influência do estresse hídrico nos parâmetros de crescimento, acúmulo de nitrogênio e produtividade em diferentes variedades de cana-de-açúcar em Miracema, RJ. Revista Universidade Rural - Série Ciências Exatas e da Terra, Seropédica, v.24, n.2, p.18-25, 2004. 